\title{
Some Results of half Canonical Cosine and Sine Transforms
}

\author{
S. B. Chavhan, V. C. Borkar \\ Department of mathematics Yeshwant Mahavidyalaya Nanded-432016
}

\begin{abstract}
In this paper, have study new results of half canonical cosine and sine transforms of generalized function.
\end{abstract}
Keyword: Canonical transform, half canonical cosine transform, half canonical sine transform, testing function space.

1. Introduction: Now a days, fractional integral transforms play an important role in signal processing, image reconstruction, pattern recognition, accostic signal processing [1],[2]. A new generalized integral transform was obtained by Zayed[4]. Bhosale and Chaudhary [3], had extended fractional Fourier transform to the distribution of compact support. Definitions half canonical cosine and half canonical sine transform as

$$
\{\operatorname{HCCTf}(t)\}(s)=\sqrt{\frac{2}{\pi i b}} e^{\frac{i}{2}\left(\frac{d}{b}\right) s^{2}} \int_{0}^{\infty} \cos \left(\frac{s}{b} t\right) e^{\frac{i}{2}\left(\frac{a}{b}\right) t^{2}} \quad \text { for } b \neq 0
$$

And

$$
\{\operatorname{HCCTf}(t)\}(s)=\sqrt{\frac{2}{\pi i b}} e^{\frac{i}{2}\left(\frac{d}{b}\right) s^{2}} \int_{0}^{\infty}(-i) \sin \left(\frac{s}{b} t\right) e^{\frac{i}{2}\left(\frac{a}{b}\right) t^{2}} f(t) \text { for } b \neq 0
$$

Notation and terminology as per Zemanian[5],[6].This paper is organized as section 2 definition of testing function space .Section 3 definition of half canonical cosine transform and its inversion theorem. Section 4 definition of half canonical sine transform and its inversion theorem. Lastly conclusion is stated.

\section{Definition of testing function space $E$ :}

An infinitely differentiable complex valued function $\phi$ on $R^{n}$ belongs to $E\left(R^{n}\right)$, if for each compact set. $I \subset s_{a}$ where $s_{a}=\left\{t: \in R^{n}, \quad|t| \leq a, a>0\right\}$ and for $k \in R^{n}$,

$$
\gamma_{E, k} \phi(t)=\sup _{t \in I}\left|D^{k} \phi(t)\right|<\infty \quad \mathrm{k}=0,1,2,3 \ldots . .
$$

Note that space $E$ is complete and a Frechet space,let $E^{\prime}$ denotes the dual space of $E$

\section{Definition of half canonical cosine transform:}

Half canonical cosine transform of $f(t)$ is given by

$$
\begin{array}{cc}
\{\operatorname{HCCTf}(t)\}(s)=\sqrt{\frac{2}{\pi i b}} e^{\frac{i}{2}\left(\frac{d}{b}\right) s^{2}} \int_{0}^{\infty} \cos \left(\frac{s}{b} t\right) e^{\frac{i}{2}\left(\frac{a}{b}\right) t^{2}} & \text { for } b \neq 0 \\
=\sqrt{d} \cdot e^{\frac{i}{2} c d s^{2}} f(d . s) & \text { for } \mathrm{b}=0
\end{array}
$$

$\quad \quad \quad K_{H C}(t, s)=\sqrt{\frac{2}{\pi i b}} e^{\frac{i}{2}\left(\frac{d}{b}\right)^{s^{2}}} \cos \left(\frac{s}{b} t\right) e^{\frac{i}{2}\left(\frac{a}{b}\right) t^{2}}$

Hence half canonical cosine transform of $f(t)$ is defined as

$$
\{\operatorname{HCCT} f(t)\}(s)=\left\langle f(t), K_{H C} f(t, s)\right\rangle
$$


Since the range of integration for the half canonical cosine transform is just $[0, \infty]$ and not $(-\infty, \infty)$ using half canonical cosine transform is more convenient than using the canonical transform to deal with the even function.

\subsection{Inverse of half canonical cosine transform:}

Theorem 3.1: If $\{H C C T f(t)\}(s)$ is the half canonical cosine transform of $f(t)$ then $f(t)=e^{\frac{-i}{2}\left(\frac{a}{b}\right) t^{2}} \sqrt{\frac{\pi i}{2 b}} \int_{0}^{\infty} \cos \left(\frac{s}{b} t\right) e^{\frac{i}{2}\left(\frac{d}{b}\right) s^{2}}\{\operatorname{HCCT} f(t)\}(s) d s$

Proof: Using definition of half canonical cosine transform as

$$
\begin{aligned}
\{\operatorname{HCCTf}(t)\}(s) & =\sqrt{\frac{2}{\pi i b}} e^{\frac{i}{2}\left(\frac{d}{b}\right) s^{2}} \int_{0}^{\infty} \cos \left(\frac{s}{b} t\right) e^{\frac{i}{2}\left(\frac{a}{b}\right) t^{2}} \\
f(s) \sqrt{\frac{\pi i b}{2}} e^{\frac{-i}{2}\left(\frac{d}{b}\right) s^{2}} & =\int_{0}^{\infty} \cos \left(\frac{s}{b} t\right) e^{\frac{i}{2}\left(\frac{a}{b}\right) t^{2}} f(t) d t
\end{aligned}
$$

where, $f(s)=\{\operatorname{HCST} f(t)\}(s)$

$$
\begin{gathered}
\therefore g(t)=e^{\frac{i}{2}\left(\frac{a}{b}\right) t^{2}} f(t) \text { and } C_{1}(s)=\int_{0}^{\infty} g(t) \cos \left(\frac{s}{b} t\right) d t \\
C_{1}(s)=\{H C C T g(t)\}\left(\frac{s}{b}\right)
\end{gathered}
$$

where $\{\operatorname{HCCTg}(t)\}\left(\frac{s}{b}\right)$ is half canonical cosine transform of $g(t)$. Half canonical cosine transform $g(t)$ with $\operatorname{argument} \frac{s}{b}=\eta$ and $\frac{d s}{b}=d \eta$

$$
C_{1}(s)=\{\operatorname{HCCTg}(t)\}(\eta)
$$

By invoking inversion formula we get

$$
\begin{aligned}
g(t)= & \int_{0}^{\infty} \cos (\eta t) C_{1}(s) d \eta \\
& g(t)=\int_{0}^{\infty} \cos \left(\frac{s}{b} t\right) C_{1}(s) \frac{d s}{b} \\
& f(t) e^{\frac{i}{2}\left(\frac{a}{b}\right) t^{2}}=\frac{1}{b} \int_{0}^{\infty} \cos \left(\frac{s}{b} t\right) \frac{\sqrt{\pi i b}}{\sqrt{2}} f(s) e^{\frac{-i}{2}\left(\frac{d}{b}\right) s^{2}} d s \\
f(t) e^{\frac{i}{2}\left(\frac{a}{b}\right) t^{2}} & =\frac{\sqrt{\pi i b}}{\sqrt{2}} \times \frac{1}{b} \int_{0}^{\infty} \cos \left(\frac{s}{b} t\right) e^{\frac{-i}{2}\left(\frac{d}{b}\right) s^{2}} f(s) d s \\
f(t)=e^{\frac{-i}{2}\left(\frac{a}{b}\right) t^{2}} & \frac{\sqrt{\pi i}}{\sqrt{2 b}} \int_{0}^{\infty} \cos \left(\frac{s}{b} t\right) e^{\frac{-i}{2}\left(\frac{d}{b}\right) s^{2}}\{\operatorname{HCCT} f(t)\}(s) d s
\end{aligned}
$$

\section{Definition half canonical sine transform:}

Half canonical sine transform of $f(t)$ is given by

$$
\begin{aligned}
\{\operatorname{HCCTf}(t)\}(s) & =\sqrt{\frac{2}{\pi i b}} e^{\frac{i}{2}\left(\frac{d}{b}\right) s^{2}} \int_{0}^{\infty}(-i) \sin \left(\frac{s}{b} t\right) e^{\frac{i}{2}\left(\frac{a}{b}\right) t^{2}} f(t) & & \text { for } b \neq 0 \\
& =\sqrt{d} \cdot e^{\frac{i}{2} c d s^{2}} f(d . s) & & \text { for } \mathrm{b}=0
\end{aligned}
$$


where, $\quad K_{H S}(t, s)=\sqrt{\frac{2}{\pi i b}} e^{\frac{i}{2}\left(\frac{d}{b}\right) s^{2}}(-i) \sin \left(\frac{s}{b} t\right) e^{\frac{i}{2}\left(\frac{a}{b}\right) t^{2}}$

Hence half canonical sine transform of $f(t)$ is defined as

$$
\{H C S T f(t)\}(s)=\left\langle f(t), K_{H S} f(t, s)\right\rangle
$$

Since the range of integration for the half canonical sine transform is just $[0, \infty]$ and not $(-\infty, \infty)$ using half canonical sine transform is more convenient than using the canonical transform to deal with the even function.

\subsection{Inverse of half canonical sine transform:}

Theorem4.1: If $\{\operatorname{HCST} f(t)\}(s)$ is the half canonical sine transform of $f(t)$ then $f(t)=-i \sqrt{\frac{\pi i}{2 b}} e^{\frac{-i}{2}\left(\frac{a}{b}\right) t^{2}} \int_{0}^{\infty} \sin \left(\frac{s}{b} t\right) e^{\frac{-i}{2}\left(\frac{d}{b}\right) s^{2}}\{\operatorname{HCST} f(t)\}(s) d s$

Proof: Using definition of half canonical sine transform as

$$
\begin{aligned}
& \{\operatorname{HCST} f(t)\}(s)=\sqrt{\frac{2}{\pi i b}} e^{\frac{i}{2}\left(\frac{d}{b}\right) s^{2}} \int_{0}^{\infty}(-i) \sin \left(\frac{s}{b} t\right) e^{\frac{i}{2}\left(\frac{a}{b}\right) t^{2}} f(t) d t \\
& F(s)=(-i) \sqrt{\frac{2}{\pi i b}} e^{\frac{i}{2}\left(\frac{d}{b}\right) s^{2}} \int_{0}^{\infty} \sin \left(\frac{s}{b} t\right) e^{\frac{i}{2}\left(\frac{a}{b}\right) t^{2}} f(t) d t
\end{aligned}
$$

where, $\quad f(s)=\{\operatorname{HCST} f(t)\}(s)$

$$
\begin{aligned}
& F(s) \sqrt{\frac{\pi i b}{2}} e^{\frac{-i}{2}\left(\frac{d}{b}\right) s^{2}}=(-i) \int_{0}^{\infty} \sin \left(\frac{s}{b} t\right) e^{\frac{i}{2}\left(\frac{a}{b}\right) t^{2}} f(t) d t \\
& C_{1}(s)=(-i) \int_{0}^{\infty} \sin \left(\frac{s}{b} t\right) g(t)
\end{aligned}
$$

where, $\quad C_{1}(s)=F(s) \sqrt{\frac{\pi i b}{2}} e^{\frac{-i}{2}\left(\frac{d}{b}\right) s^{2}}$

$\therefore g(t)=e^{\frac{i}{2}\left(\frac{a}{b}\right) t^{2}} f(t)$ and $\quad C_{1}(s)=\{\operatorname{HCSTg}(t)\}\left(\frac{s}{b}\right)$

where $\{\operatorname{HCSTg}(t)\}\left(\frac{s}{b}\right)$ is half canonical sine transform of $g(t)$. Half canonical sine transform $g(t)$ with argument $\frac{s}{b}=\eta$ and $\frac{d s}{b}=d \eta$

$$
C_{1}(s)=\{\operatorname{HCSTg}(t)\}(\eta)
$$

By inversion formula we get

$$
\begin{gathered}
g(t)=(-i) \int_{0}^{\infty} \sin (\eta t) C_{1}(s) d \eta \\
g(t)=(-i) \int_{0}^{\infty} \sin \left(\frac{s}{b} t\right) C_{1}(s) \frac{d s}{b} \\
g(t)=(-i) \int_{0}^{\infty} \sin \left(\frac{s}{b} t\right) \sqrt{\frac{\pi i b}{2}} f(s) e^{\frac{-i}{2}\left(\frac{d}{b}\right) s^{2}} \frac{d s}{b} \\
f(t)=(-i) \sqrt{\frac{\pi i}{2 b}} e^{\frac{-i}{2}\left(\frac{a}{b}\right) t^{2}} \int_{0}^{\infty} \sin \left(\frac{s}{b} t\right) e^{\frac{-i}{2}\left(\frac{d}{b}\right) s^{2}} f(s) d s
\end{gathered}
$$




$$
f(t)=(-i) \sqrt{\frac{\pi i}{2 b}} e^{\frac{-i}{2}\left(\frac{a}{b}\right) t^{2}} \int_{0}^{\infty} \sin \left(\frac{s}{b} t\right) e^{\frac{-i}{2}\left(\frac{d}{b}\right) s^{2}}\{\operatorname{HCST} f(t)\}(s) d s
$$

\section{Conclusion:}

In this paper half canonical cosine and half canonical sine transforms is generalized in the form the distributional sense, we have inversion theorem for this transforms are proved.

\section{References:}

[1] Alieva Tatiana. and Bastianas Martin J.,: "On Fractional Fourier transform moments," IEEE signal processing letters, Vol. 7, No.11, Nov. 2000.

[2] Alieva Tatiana. and Bastiaans Martian J.,: "Wigner Distribution and Fractional Fourier Transform for 2Dimentional Symmetric Beams", JOSA A, Vol.17, No.12, P.2319 - 2323, Dec.2000.

[3] Bhosale B.N. and Choudhary M.S.,: "Fractional fourier transform of distributions of,compact support,"Bull.Cal.Math.Soc.,Vol.94,No.5,349-358,2002.

[4] Zayed A. : "A convolution and product theorem for the fractional Fourier transform", IEEE sign. proc. letters Vol.5, No.4, (1998), 101-103.

[5] Zemanian A.H., : "Distribution theory and transform analysis", McGraw Hill, New York, (1965).

[6] Zemanian A.H., : "Generalized Integral Transform”, Inter Science Publisher's New York, (1968). 